Research Article

\title{
Nontyphoidal Salmonella and Their Antimicrobial Susceptibility among Diarrheic Patients Attending Private Hospitals in Addis Ababa, Ethiopia
}

\author{
Ruhama Kebede, Haile Alemayehu, Girmay Medhin $(\mathbb{D}$, and Tadesse Eguale $\mathbb{1}$ \\ Aklilu Lemma Institute of Pathobiology, Addis Ababa University, P.O. Box 1176, Addis Ababa, Ethiopia \\ Correspondence should be addressed to Tadesse Eguale; tadesse.eguale@aau.edu.et
}

Received 9 April 2021; Revised 7 July 2021; Accepted 7 September 2021; Published 18 September 2021

Academic Editor: Mohamed Salah Abbassi

Copyright (c) 2021 Ruhama Kebede et al. This is an open access article distributed under the Creative Commons Attribution License, which permits unrestricted use, distribution, and reproduction in any medium, provided the original work is properly cited.

\begin{abstract}
Nontyphoidal Salmonella (NTS) is one of the major causes of bacterial foodborne infection. It is mainly manifested by self-limiting gastroenteritis in healthy individuals but can also cause severe complications including blood stream infection and mortality. The emergence of multidrug-resistant strains of Salmonella is becoming a global public health concern. This study is aimed at estimating the prevalence of Salmonella, identifying serotypes involved, and investigating antimicrobial susceptibility of the isolates among diarrheic patients attending private hospitals in Addis Ababa. We collected a total of 298 stool samples from diarrheic patients attending five private hospitals in Addis Ababa and isolated Salmonella according to standard microbiological techniques; the isolates were serotyped using slide agglutination and microplate agglutination techniques. Antimicrobial susceptibility test of the isolates was carried out using Kirby-Bauer disc diffusion assay according to Clinical Laboratory Standards Institute guidelines. Fourteen stool samples (4.7\%) were positive for Salmonella, and Salmonella Kiambu was the most dominant serovar $(n=7,50 \%)$ followed by $S$. Saintpaul $(n=4,28.6 \%)$ and $S$. Haifa $(n=2,14.3 \%)$. Three $(21.4 \%)$ of the isolates were resistant to sulfisoxazole and tetracycline each and $2(14.3 \%)$ to ampicillin. Resistance to two antimicrobials was detected only in $2(14.3 \%)$ of the isolates, and none of the isolates were resistant to more than two antimicrobials. In conclusion, the current study showed low prevalence of NTS in diarrheic patients attending private hospitals in Addis Ababa. Although multidrug resistance to several antimicrobials was not detected in the isolates, prudent use of antimicrobials is recommended to guaranty the long-term use of the available antimicrobials.
\end{abstract}

\section{Introduction}

Infection with NTS causes significant morbidity and mortality in humans and animals globally. Infection with NTS causes symptoms that can range from mild self-limiting gastroenteritis to severe invasive septicemia and can occasionally be fatal. Most of the infections caused by nontyphoidal Salmonella serovars are characterized by self-limiting acute gastroenteritis and diarrhea [1-3]. In addition to illness and death inflicted by NTS serovars, it was estimated to cause 4 million disability adjusted life years (DALYS) accounting for $22.2 \%$ of DALYS due to diarrhea causing agents that leads to huge economic loss [4]. In human, NTS infection usually causes foodborne outbreaks [3]. The common sources of infections are various animal products such as poultry, dairy, and pork [5]. Salmonella can also be transmitted from person to person, domestic animals like dogs, cats, and rodents, and consumption of contaminated products like sprouts, tomatoes, fruits, peanuts, and spinaches [5-7].

For decades, antimicrobials have reduced morbidity and mortality due to various bacterial infections worldwide. However, easy access to antimicrobials without prescription and extensive misuse both in public health and animal health sectors has led to emergence and spread of resistant strains of pathogenic bacteria $[8,9]$. The emergence and rapid spread of antimicrobial resistance (AMR) in Salmonella has become a worldwide concern. In addition to 
resistance to first-line antimicrobials like third-generation cephalosporins and fluoroquinolones, plasmid borne resistance to colistin (the last resort antimicrobial for treatment of multidrug gram negative bacterial infections) mediated by different variants of $\mathrm{mcr}$ gene has been reported in Salmonella isolates from different countries [10]. An increasing number of treatment failures are often linked to multidrug resistant (MDR) Salmonella strains [11]. MDR Salmonella isolates have been associated with high risk of invasive infection, an increased risk of death, and prolonged illness as compared to infections caused by susceptible strains [12].

In Ethiopia, there are studies that indicated occurrence of Salmonella and high rate of antimicrobial resistance in both public health and veterinary sectors. For example, a study that investigated diarrheic patients attending health care facility in Gondar reported that 1.2\% (4/372) of the stool samples were positive for Salmonella [13]. The isolates detected in this study were resistant to tetracycline (100\%), amoxicillin (100\%), and ampicillin (75\%). Other study conducted in Nekemte referral hospital reported Salmonella in $2.1 \%(9 / 422)$ of the diarrheic study participants, and all of the isolates were resistant to amoxicillin [14]. In a study conducted in Southern Ethiopia, Salmonella was detected in $1.0 \%(2 / 204)$ of diarrheic patients and both isolates were resistant to ampicillin and gentamicin [15].

Most of the previous studies in Ethiopia were conducted on patients attending public health facilities, and little data is available on prevalence and antimicrobial susceptibility of Salmonella isolates from patients attending private health facilities. Due to difference in income, educational status and exposure to different predisposing factors, rate of Salmonella infection and antimicrobial susceptibility status of isolates might be different in people attending private health facilities compared to those attending government-owned health facilities. Estimating the prevalence of Salmonella among diarrheic patients and establishing antimicrobial susceptibility status of isolates combined with investigation of susceptibility of isolates to antimicrobials are of paramount importance to advice clinicians on appropriate management of Salmonella infection. Results from such study will also strengthen epidemiological knowledge of the disease that could help policy makers while planning interventions for the at-risk populations.

The objectives of this study were therefore (a) to estimate the prevalence of Salmonella among diarrheic patients attending private hospitals in Addis Ababa and to identify serotypes involved, (b) to investigate factors that can potentially be associated with Salmonella positivity, and (c) to assess antimicrobial susceptibility of Salmonella isolates.

\section{Materials and Methods}

2.1. Study Area, Context of the Study, and Study Design. The current study was conducted in Addis Ababa, the capital city of Ethiopia. The city lies at an elevation of 2,355 meters above sea level (m.a.s.l) and located at $9^{\circ} 1^{\prime} 48^{\prime \prime}$ North and $38^{\circ} 44^{\prime} 24^{\prime \prime}$ East, and it has a subtropical highland climate, with average annual temperature of $16.3^{\circ} \mathrm{C}$ and $1089 \mathrm{~mm}$ annual rainfall. Administratively, Addis Ababa was divided into 10 subcities, namely, Addis Ketema, Akaki-Kality, Arada, Bole, Gulele, Kirkos, Kolfe-Keranyo, Lideta, Nifas Silk-Lafto, and Yeka, when this study was conducted. According to World Population Review, population of Addis Ababa was estimated to be 4,591,983 in 2019 with average population density of 5,165 individuals per square kilometer [16].

Residents of Addis Ababa receive health services both from health institutions owned by the government and by private sectors. According to Addis Ababa City Administration Food, Medicine and Health Care Administration and Control Authority, there were more than 22 higher private hospitals in Addis Ababa in 2018 (personal communication). Although seven private hospitals were randomly selected, only five hospitals located in 4 of the 10 subcities in Addis Ababa agreed to participate in the study. Hence, diarrheic patients were recruited from these five private hospitals, namely, Bethezatha located in Kirkos subcity, Ethio Tebib in Addis Ketema subcity, iCMC and Migbaresenay Hospitals located in Yeka subcity, and Teklehaimanot Hospital located in Arada subcity. A cross-sectional study design was used to estimate prevalence of Salmonella in diarrheic patients by examining human stool samples and to determine antimicrobial susceptibility of the isolates.

2.2. Stool Sample Collection and Interviewing of Study Participants. Stool sample collection and patient interviews were conducted from September 2018 to June 2019. Two hundred and ninety-eight stool samples were collected from diarrheic patients attending five private hospitals during the study period. Screw-caped clean plastic containers were used for the collection of stool samples. The samples were transported to Microbiology Laboratory of Aklilu Lemma Institute of Pathobiology in an icebox within 3-4 hours of collection. Various patient-related data including age, sex, history of recent antimicrobial usage, marital status, occupation, educational status, hand washing habit, and habit of consumption of raw vegetables and raw meat were collected directly by interviewing the study participants using structured questionnaire to investigate possible factors associated with occurrence of Salmonella and antimicrobial resistance.

2.3. Isolation, Identification, and Serotyping of Salmonella. Salmonella isolation was conducted according to Global Foodborne Infections Network, laboratory protocol [17]. Briefly, $1 \mathrm{~g}$ of stool sample was suspended in $9 \mathrm{ml}$ of buffered peptone water (Himedia, India) and incubated for 24 hours at $37^{\circ} \mathrm{C}$. Then, $100 \mu \mathrm{l}$ of the suspension was transferred to $10 \mathrm{ml}$ of Rappaport Vassiliadis enrichment broth (RVB), (Oxoid, UK) and incubated for $24 \mathrm{~h}$ at $42^{\circ} \mathrm{C}$. Suspension of $1 \mathrm{ml}$ of each sample was also transferred to $9 \mathrm{ml}$ of Muller-Kauffmann-Tetrathionate broth (Himedia, India) and Selanite-F broth (Becton-Dickinson, USA) and incubated for $24 \mathrm{~h}$ at $37^{\circ} \mathrm{C}$. Samples from these three enrichment broths were streaked on to Xylose-Lysine Deoxycholate Agar (Himedia, India), and the plates were incubated at $37^{\circ} \mathrm{C}$ for 24-48 hours.

Biochemical tests were conducted for presumptive Salmonella colonies using Urea, Triple Sugar Iron Agar, Lysine 
Iron Agar, and Citrate as described elsewhere. Typical Salmonella colonies were confirmed using genus specific PCR as described previously [18]. Serotyping of Salmonella isolates was conducted at the National Microbiology Laboratory, Office International des Epizooties (OIE) Salmonella Reference Laboratory, Public Health Agency of Canada. Somatic $(\mathrm{O})$ antigens were determined using slide agglutination tests whereas flagellar antigens using microplate agglutination technique $[19,20]$.

2.4. Antimicrobial Susceptibility Testing. Salmonella isolates were investigated for susceptibility to antimicrobials commonly prescribed for treatment of Salmonella or other related enteric pathogens using the Kirby-Bauer disk diffusion method according to Clinical Laboratory Standards Institute guideline [21]. The following antimicrobials (Sensi-Discs, Becton, Dickinson and Company, Loveton, USA) and disc potencies $(\mu \mathrm{g})$ were used with different concentration, amikacin $(30 \mu \mathrm{g})$, amoxicillin+clavulanic acid $(20 / 10 \mu \mathrm{g})$, ampicillin $(10 \mu \mathrm{g})$, ceftriaxone $(30 \mu \mathrm{g})$, cephalothin $(30 \mu \mathrm{g})$, chloramphenicol $(30 \mu \mathrm{g})$, ciprofloxacin $(5 \mu \mathrm{g})$, gentamicin $(10 \mu \mathrm{g})$, streptomycin $(10 \mu \mathrm{g})$, sulfisoxazole $(1000 \mu \mathrm{g})$, sulfamethoxazole+trimethoprim $(23.75 / 1.25 \mu \mathrm{g})$, and tetracycline $(30 \mu \mathrm{g})$. Escherichia coli ATCC 25922 was used for a quality control.

2.5. Ethical Consideration. The conduct of the study was approved for its ethical issues by the Institutional Review Board of Aklilu Lemma Institute of Pathobiology, Addis Ababa University (Minutes Ref No.: ALIPB/IRB/014/2011/19). Prior to sample collection, verbal consent was obtained from the study participants. Study participants were informed about the overall objective of the study, the voluntary participation, and their rights to withdraw from the study if they want to do so.

2.6. Data Analysis. Summarized findings were presented in Tables. The association between background characteristics of study participants and occurrence of Salmonella was assessed using exact test because some cell frequencies were small violating the assumptions required by Pearson-chisquare test. Associations were reported as being statistically significant whenever $p$ value was less than 0.05 . Statistical Package for Social Sciences (SPSS, version 20.0) was used to facilitate analysis of the data.

\section{Results}

3.1. Characteristics of Study Participants. Out of 298 study participants, $59 \%$ were male, $62.3 \%$ were married, $41.9 \%$ were college/university graduates or studying, and $24.9 \%$ were business persons (Table 1).

3.2. Prevalence of Salmonella. The prevalence of Salmonella was $4.0 \%$ among males and $5.7 \%$ among female diarrheic patients. The isolation of Salmonella was highest (8.4\%) in the diarrheic patients of age range of 31-45 years although the difference was not statistically significant compared to other age group ( $p$ value $=0.3$ ). Salmonella was detected in $5 \%(9 / 178)$ of those who have the habit of consuming raw vegetables and in $5.7 \%(5 / 87)$ of those who do not have the habit of consuming raw vegetables (Table 2).

3.3. Behavior of Study Participants on the Use of Antimicrobials. Out of 241 diarrheic patients involved in this study, 9 (3.7\%) reported sharing prescribed antimicrobials with someone else, 181 (78.4\%) participants were unaware about antimicrobial resistance, 76 (31.5\%) did not complete prescribed dose in a single session of therapy, and 110 (41.5\%) participants reported that they get antimicrobials from pharmacy without prescription for various ailments (Table 3).

3.4. Serovar Distribution and Antimicrobial Susceptibility of Salmonella Isolated from Diarrheic Patients. Four different Salmonella serovars were detected in the current study. Salmonella Kiambu $(n=7)$ was the most frequently isolated followed by $S$. Saintpaul $(n=4), S$. Haifa $(n=2)$, and $S$. Enteritidis $(n=1)$. The findings of antimicrobial susceptibility test showed that 3 isolates (21.4\%) were resistant to sulfisoxazole, 3 isolates $(21.4 \%)$ were resistant to tetracycline, and 2 isolates (14.3\%) were resistant to ampicillin. Three out of 7 S. Kiambu isolates (42.9\%) were resistant to sulfisoxazole. Resistance to tetracycline was recorded in the three serovars except $S$. Enteritidis. All Salmonella isolates were susceptible to amikacin, cephalothin, amoxicillin+clavulanic acid, chloramphenicol, ceftriaxone, ciprofloxacin, gentamicin, streptomycin, and sulfamethoxazole+trimethoprim (Table 4).

Coresistance to ampicillin and tetracycline was detected only in $14.3 \%(2 / 14)$ of the isolates, one from $S$. Kiambu and the other from $S$. Saintpaul. Majority of the isolates were susceptible to most of the antimicrobials investigated, and MDR to over two antimicrobials was not detected.

\section{Discussion}

In the current study, prevalence of Salmonella among diarrheic patients was $4.7 \%$ which is lower than what was reported in a previous study (7.2\%) in Addis Ababa among patients attending government-owned health centers but higher than $1.1 \%$ reported from a study in Gondar, North Ethiopia [13] and diarrheic children in Ambo town (1.2\%) [22]. Prevalence of Salmonella in the current study is in close agreement with previous reports in Addis Ababa (4.5\%) [23] and $5.4 \%$ prevalence among HIV positive and $4.5 \%$ among HIV-negative diarrheic patients in Dessie town, North Ethiopia [24]. Meta-analysis of works done in Ethiopia showed $5.7 \%$ prevalence of Salmonella in diarrheic adult patients [25]. The difference in isolation rate of Salmonella might be explained by difference in socioeconomic status of the diarrheic patients which could be expressed through difference in life style like hand washing and other hygienic practices. Since health services in private hospitals are expensive in Addis Ababa as compared to government hospitals, better ability to pay might be main driving force to attend private hospitals. Most previous studies in Ethiopia recruited study participants from outpatient clinics of government-owned health centers [24-27]. It is believed that only patients with 
TABLE 1: Background information of diarrheic patients participated in the study.

\begin{tabular}{|c|c|c|c|}
\hline Characteristics & Category & Number & Percentage \\
\hline \multirow{2}{*}{$\operatorname{Sex}(N=298)$} & Male & 176 & 59 \\
\hline & Female & 122 & 40.9 \\
\hline \multirow{5}{*}{ Age group $(N=265)$} & $0-5$ & 24 & 9.1 \\
\hline & $6-18$ & 17 & 6.4 \\
\hline & $19-30$ & 76 & 28.7 \\
\hline & $31-45$ & 95 & 35.8 \\
\hline & $>45$ & 53 & 20 \\
\hline \multirow{5}{*}{ Education $(N=265)$} & Illiterate & 20 & 7.5 \\
\hline & Underage/preschool & 24 & 9 \\
\hline & Primary school (1-8) & 36 & 13.6 \\
\hline & Secondary school (9-12) & 74 & 27.9 \\
\hline & College/university & 111 & 41.9 \\
\hline \multirow{2}{*}{ Marital status $(N=265)$} & Single & 100 & 37.7 \\
\hline & Married & 165 & 62.3 \\
\hline \multirow{4}{*}{ No. of children $(N=265)$} & $1-3$ & 100 & 37.7 \\
\hline & $4-6$ & 37 & 14 \\
\hline & $>6$ & 10 & 3.8 \\
\hline & None & 118 & 44.5 \\
\hline \multirow{6}{*}{ Occupation $(N=265)$} & Businessmen/women & 66 & 24.9 \\
\hline & Student/underage & 56 & 21.1 \\
\hline & Governmental & 55 & 20.8 \\
\hline & Private & 44 & 16.6 \\
\hline & Housewife & 34 & 12.8 \\
\hline & Other & 10 & 3.8 \\
\hline \multirow{5}{*}{ Place of sample collection $(N=298)$} & Bethezatha Hospital & 59 & 19.8 \\
\hline & Ethio Tebib Hospital & 59 & 19.8 \\
\hline & iCMC Hospital & 15 & 5 \\
\hline & Migbaresenay Hospital & 8 & 2.7 \\
\hline & Teklehaimanot Hospital & 157 & 52.7 \\
\hline
\end{tabular}

good income can afford to visit private hospitals. In addition, season of sample collection, geographic location, and habit of consuming raw vegetables and raw animal products and illness due to other enteric pathogens could affect the rate of detection of Salmonella from diarrheic patients.

In the current study, none of the patient-related factors were shown to have statistically significant association with detection of Salmonella from stool of diarrheic patients unlike previous studies where consumption of raw vegetables and stool being watery [26], and poor hand washing practices and consumption of raw/uncooked foods were associated with detection of Salmonella in diarrheic patients [28]. The reason why we did not observe such association in this study could be due to small sample size and better practices of cleaning raw vegetables before consumption among study participants.

The most prevalent Salmonella serovar in the current study was $S$. Kiambu followed by $S$. Saintpaul. In the previous studies conducted in Addis Ababa, S. Typhimurium was the dominant serotype followed by $S$. Virchow [26] whereas these serovars were not isolated in the current study. Salmonella Concord and S. Typhimurium were reported to be the dominant serovars in diarrheic patients in Addis Ababa in other previous studies [27, 29]. Previous studies in other subSaharan African countries also showed dominance of $S$. Typhimurium [30, 31]. Different serovars of Salmonella are known to be commonly detected in a given area at different times depending on the type of serovars of Salmonella circulating in food animals and food sources in a study area. Epidemiology of NTS is shown to be characterized by temporal variation in dominance of certain Salmonella serovars at a time followed by replacement with other serovars [32].

The present study revealed that irrational use of antimicrobials such as failure to finish the whole course of antimicrobials, taking antimicrobials without prescription, and sharing antimicrobials with relatives and or friends were the commonly observed behavior of the study participants. This finding agrees with previous studies conducted in 
TABLe 2: Prevalence of Salmonella among diarrheic patients attending private hospitals stratified by selected background characteristics.

\begin{tabular}{|c|c|c|c|c|c|}
\hline \multirow{2}{*}{ Characteristics } & \multirow{2}{*}{ Category } & \multicolumn{3}{|c|}{ Salmonella status } & \multirow{2}{*}{$p$ value } \\
\hline & & No. examined & No. positive & Percent & \\
\hline \multirow{2}{*}{$\operatorname{Sex}(N=298)$} & Male & 176 & 7 & 4.0 & \multirow{2}{*}{0.6} \\
\hline & Female & 122 & 7 & 5.7 & \\
\hline \multirow{5}{*}{ Age group $(N=265)$} & $0-5$ & 24 & 1 & 4.2 & \multirow{5}{*}{0.3} \\
\hline & $6-18$ & 17 & 1 & 5.9 & \\
\hline & $19-30$ & 76 & 1 & 1.3 & \\
\hline & $31-45$ & 95 & 8 & 8.4 & \\
\hline & $>45$ & 53 & 3 & 5.7 & \\
\hline \multirow{5}{*}{ Educational status $(N=265)$} & Illiterate & 16 & 1 & 6.3 & \multirow{5}{*}{0.4} \\
\hline & Underage/preschool & 25 & 3 & 12.0 & \\
\hline & Primary school (1-8) & 40 & 3 & 7.5 & \\
\hline & Secondary school (9-12) & 74 & 3 & 4.0 & \\
\hline & College/university & 110 & 4 & 3.6 & \\
\hline \multirow{6}{*}{ Occupation $(N=265)$} & Business person & 66 & 4 & 6.0 & \multirow{6}{*}{0.6} \\
\hline & Student/underage & 56 & 2 & 3.6 & \\
\hline & Governmental & 55 & 3 & 5.5 & \\
\hline & Private & 44 & 1 & 2.3 & \\
\hline & Housewife & 34 & 4 & 11.8 & \\
\hline & Others & 10 & 0 & 0.0 & \\
\hline \multirow{2}{*}{${ }^{*}$ handwashing habit after using toilet $(N=241)$} & Yes & 213 & 10 & 4.7 & \multirow{2}{*}{0.2} \\
\hline & No & 28 & 3 & 10.7 & \\
\hline \multirow{2}{*}{ Consumption of raw vegetables $(N=265)$} & Yes & 178 & 9 & 5.0 & \multirow{2}{*}{0.8} \\
\hline & No & 87 & 5 & 5.7 & \\
\hline \multirow{2}{*}{ Consumption of raw meat $(N=265)$} & Yes & 175 & 12 & 6.9 & \multirow{2}{*}{0.2} \\
\hline & No & 90 & 2 & 2.2 & \\
\hline \multirow{4}{*}{ Stool consistency $(N=298)$} & Loose & 168 & 10 & 6.0 & \multirow{4}{*}{0.4} \\
\hline & Watery & 82 & 2 & 2.4 & \\
\hline & Mucoid & 36 & 1 & 2.8 & \\
\hline & Bloody & 12 & 1 & 8.3 & \\
\hline Total & & 298 & 14 & 4.7 & \\
\hline
\end{tabular}

*One of the Salmonella positive study participants did not respond to this question.

TABle 3: Antimicrobial usage behavior and practices of study participants.

\begin{tabular}{|c|c|c|c|}
\hline Characteristics/question of interest & Response category & Number & Percentage \\
\hline $\begin{array}{l}\text { Sharing prescribed antimicrobial with someone and vice } \\
\text { versa }(N=241)\end{array}$ & Yes & 9 & 3.7 \\
\hline Aware about antimicrobial resistance $(N=241)$ & Yes & 52 & 21.6 \\
\hline \multirow{3}{*}{ From where do you usually get antimicrobial drugs $(N=265)$} & From pharmacy with physician prescription & 147 & 55.5 \\
\hline & Without prescription from pharmacy & 110 & 41.5 \\
\hline & With/without prescription & 8 & 3.0 \\
\hline Do you always finish prescribed antimicrobials $(N=241)$ & Yes & 165 & 68.5 \\
\hline
\end{tabular}

Ethiopia [33] and elsewhere [34]. Such irrational use of antimicrobials, particularly purchase of antimicrobials without prescription, low level of finishing prescribed antimicrobials, and low level of awareness about antimicrobial resistance contributes to emergence and spread of antimicrobial resistance [35]. Hence, special attention should be paid to increase awareness of the community on rational use of antimicrobials. 
TABLE 4: Antimicrobial susceptibility profile of Salmonella serovars isolated from stool of diarrheic patients.

\begin{tabular}{|c|c|c|c|c|c|}
\hline Antimicrobials tested & $\begin{array}{l}\text { S. Enteritidis }(n=1) \\
\text { No. }(\%) \text { of resistant }\end{array}$ & $\begin{array}{c}\text { S. Haifa }(n=2) \\
\text { No. }(\%) \text { of resistant }\end{array}$ & $\begin{array}{l}\text { S. Kiambu }(n=7) \\
\text { No. }(\%) \text { of resistant }\end{array}$ & $\begin{array}{l}\text { S. Saintpaul }(n=4) \\
\text { No. }(\%) \text { of resistant }\end{array}$ & $\begin{array}{c}\text { Total No. (\%) } \\
\text { of resistant }\end{array}$ \\
\hline Amoxicillin+clavulanic acid & $0(0)$ & $0(0)$ & $0(0)$ & $0(0)$ & $0(0)$ \\
\hline Ampicillin & $0(0)$ & $0(0)$ & $1(14.3)$ & $1(25.0)$ & $2(14.3)$ \\
\hline Amikacin & $0(0)$ & $0(0)$ & $0(0)$ & $0(0)$ & $0(0)$ \\
\hline Chloramphenicol & $0(0)$ & $0(0)$ & $0(0)$ & $0(0)$ & $0(0)$ \\
\hline Cephalothin & $0(0)$ & $0(0)$ & $0(0)$ & $0(0)$ & $0(0)$ \\
\hline Ciprofloxacin & $0(0)$ & $0(0)$ & $0(0)$ & $0(0)$ & $0(0)$ \\
\hline Ceftriaxone & $0(0)$ & $0(0)$ & $0(0)$ & $0(0)$ & $0(0)$ \\
\hline Sulfisoxazole & $0(0)$ & $0(0)$ & $3(42.9)$ & $0(0)$ & $3(21.4)$ \\
\hline Gentamicin & $0(0)$ & $0(0)$ & $0(0)$ & $0(0)$ & $0(0)$ \\
\hline Streptomycin & $0(0)$ & $0(0)$ & $0(0)$ & $0(0)$ & $0(0)$ \\
\hline Sulfamethoxazole+trimethoprim & $0(0)$ & $0(0)$ & $0(0)$ & $0(0)$ & $0(0)$ \\
\hline Tetracycline & $0(0)$ & $1(50.0)$ & $1(14.3)$ & $1(25.00)$ & $3(21.4)$ \\
\hline
\end{tabular}

Relatively low proportions of the Salmonella isolates were resistant to sulfisoxazole, tetracycline and ampicillin in this study. Previous study in Ethiopia also showed similar low rate of resistance to tetracycline $(13.4 \%)$ and ampicillin $(14.3 \%)$ whereas high rate of resistance to sulfisoxazole (38.9\%) was reported [26]. The finding of the current study agrees with previous studies from Greece [36], Libya [37], and England [38]. The current finding is however very low compared to previous reports in Ethiopia [39, 40] where high rate of resistance was recorded in isolates from diarrheic patients and food handlers, respectively. Similarly, unlike previous studies conducted among diarrheic patients in Addis Ababa that reported 3\% resistance to ceftriaxone (third-generation cephalosporin) and $4.5 \%$ to ciprofloxacin [26], resistance to these antimicrobials was not detected among Salmonella isolates tested in the current study. This previous study reported MDR to 5 or more antimicrobials in $25.4 \%$ of the isolates. This could be either due to low sample size or relatively better understanding of the study population in the current study on rational use of antimicrobials and may not be exposed to frequent empirical antimicrobial therapy. Moreover, strains of Salmonella isolated from patients in the current study might have originated from reservoir hosts or food sources where there is minimal exposure to antimicrobials. Trend analysis of antimicrobial resistance among $S$. Typhimurium isolates from animal meat and humans in China for 20 years showed fluctuation over time [41]. The limitation of this study is low number of samples investigated and the number of samples collected was not equally distributed across each hospital due to low number of diarrheic patients coming to some of the hospitals during the study period.

\section{Conclusion}

The current study showed low prevalence of Salmonella in diarrheic patients attending private hospitals in Addis Ababa. Although MDR to several antimicrobials was not detected in the isolates, the observed resistance to some of antimicrobials suggests the need for prudent use of antimi- crobials to protect the long-term use of the available antimicrobials.

\section{Abbreviations}

AMR: Antimicrobial resistance

DALYS: Disability adjusted life years

MDR: Multidrug resistance

NTS: Nontyphoidal Salmonella

OIE: Office International des Epizooties.

\section{Data Availability}

All data generated or analyzed during this study are included in this published article.

\section{Conflicts of Interest}

The authors declare that they have no competing interests.

\section{Authors' Contributions}

RK was involved in collection of data, laboratory investigation, preparation of draft manuscript, and data analysis. HA was involved in laboratory activities. GM was involved in data analysis and edition of the draft manuscript. TE was involved in conception of the study, revising the draft manuscript, and acquisition of funding. All authors read and approved the final manuscript.

\section{Acknowledgments}

This study was funded by a joint grant by TDR, EDCTP, and the WHO Regional Office for Africa. The authors are grateful to the staff of the Salmonella Reference Laboratory, National Microbiology Laboratory at Guelph, OIE Reference Laboratory, Public Health Agency of Canada particularly, Dr. Gitanjali Arya, Bob Holtslander, and Kim Ziebell for serotyping Salmonella isolates. We also appreciate technical assistance Ms. Azeb Teklu during laboratory work. 


\section{References}

[1] N. A. Feasey, G. Dougan, R. A. Kingsley, R. S. Heyderman, and M. A. Gordon, "Invasive non-typhoidal salmonella disease: an emerging and neglected tropical disease in Africa," Lancet, vol. 379, no. 9835, pp. 2489-2499, 2012.

[2] T. T. Ao, N. A. Feasey, M. A. Gordon, K. H. Keddy, F. J. Angulo, and J. A. Crump, "Global burden of invasive nontyphoidal Salmonella disease, 2010," Emerging infectious diseases, vol. 21, no. 6, pp. 941-949, 2015.

[3] H.-M. Chen, Y. Wang, L.-H. Su, and C.-H. Chiu, "Nontyphoid Salmonella Infection: Microbiology, Clinical Features, and Antimicrobial Therapy," Pediatrics \& Neonatology, vol. 54, no. 3, pp. 147-152, 2013.

[4] WHO, WHO estimates of the global burden of foodborne diseases: foodborne disease burden epidemiology reference group 2007-2015, World Health Organization, Geneva, 2015.

[5] O. Gal-Mor, E. C. Boyle, and G. A. Grassl, "Same species, different diseases: how and why typhoidal and non-typhoidal Salmonella enterica serovars differ," Frontiers in Microbiology, vol. 5, p. 391, 2014.

[6] C. Bayer, H. Bernard, R. Prager et al., "An outbreak of Salmonella Newport associated with mung bean sprouts in Germany and the Netherlands, October to November 2011," Euro surveillance: bulletin Europeen sur les maladies transmissibles $=$ European communicable disease bulletin, vol. 19, no. 1, 2014.

[7] B. R. Jackson, P. M. Griffin, D. Cole, K. A. Walsh, and S. J. Chai, "Outbreak-associated Salmonella enterica serotypes and food commodities, United States, 1998-2008," Emerging Infectious Diseases, vol. 19, no. 8, pp. 1239-1244, 2013.

[8] F. Prestinaci, P. Pezzotti, and A. Pantosti, "Antimicrobial resistance: a global multifaceted phenomenon," Pathogens and global health, vol. 109, no. 7, pp. 309-318, 2015.

[9] D. K. Byarugaba, "Antimicrobial resistance in developing countries and responsible risk factors," International Journal of Antimicrobial Agents, vol. 24, no. 2, pp. 105-110, 2004.

[10] M. Elbediwi, Y. Li, N. Paudyal et al., "Global burden of colistinresistant bacteria: mobilized colistin resistance genes study (1980-2018)," Microorganisms, vol. 7, no. 10, p. 461, 2019.

[11] K. L. Shrestha, N. D. Pant, R. Bhandari, S. Khatri, B. Shrestha, and B. Lekhak, "Re-emergence of the susceptibility of the Salmonella spp. isolated from blood samples to conventional first line antibiotics," Antimicrobial Resistance \& Infection Control, vol. 5, no. 1, p. 22, 2016.

[12] S. Mukherjee, C. M. Anderson, R. E. Mosci et al., "Increasing frequencies of antibiotic resistant non-typhoidal Salmonella infections in Michigan and risk factors for disease," Frontiers in Medicine, vol. 6, p. 250, 2019.

[13] T. A. Demissie, F. M. Yehuala, D. M. Fetene, and G. Y. Gudeta, "Prevalence and antimicrobial susceptibility patterns of Shigella and Salmonella species among patients with diarrhea attending Gondar Town Health Institutions, Northwest Ethiopia," Science Journal of Public Health, vol. 2, no. 5, pp. 469475,2014

[14] A. Terfassa and M. Jida, "Prevalence and antibiotics susceptibility pattern of Salmonella and Shigella species among diarrheal patients attending Nekemte Referral Hospital, Oromia, Ethiopia," International journal of microbiology, vol. 2018, Article ID 9214689, 6 pages, 2018.

[15] W. Abebe, A. Earsido, S. Taye, M. Assefa, A. Eyasu, and G. Godebo, "Prevalence and antibiotic susceptibility patterns of Shigella and Salmonella among children aged below five years with diarrhoea attending Nigist Eleni Mohammed memorial hospital, South Ethiopia," BMC pediatrics, vol. 18, no. 1, p. 241, 2018.

[16] WPR, "World population review: population of Addis Ababa in 2019," 2021.

[17] E. I. Nweze and S. Ezute, "Isolation and antifungal susceptibility of Exophiala dermatitidis isolates from human stool samples in Nigeria," Mycopathologia, vol. 169, no. 3, pp. 201206, 2010.

[18] N. D. Cohen, H. L. Neibergs, E. D. McGruder et al., "Genusspecific detection of salmonellae using the polymerase chain reaction (PCR)," Journal of Veterinary Diagnostic Investigation, vol. 5, no. 3, pp. 368-371, 1993.

[19] W. H. Ewing, Edwards and Ewing's identification of Enterobacteriaceae, Elsevier, New York, 1986.

[20] C. R. Shipp and B. Rowe, "A mechanised microtechnique for Salmonella serotyping," Journal of Clinical Pathology, vol. 33, no. 6, pp. 595-597, 1980.

[21] CLSI, Performance Standards for Antimicrobial Susceptibility Testing, P. A. Wayne, Ed., C. a. L. S. Institute, 2016.

[22] W. Tosisa, A. Mihret, A. Ararsa, T. Eguale, and T. Abebe, "Prevalence and antimicrobial susceptibility of Salmonella and Shigella species isolated from diarrheic children in Ambo town," BMC pediatrics, vol. 20, no. 1, p. 91, 2020.

[23] M. Ashenafi and M. Gedebou, "Salmonella and Shigella in adult diarrhoea in Addis Ababa prevalence and antibiograms," Transactions of the Royal Society of Tropical Medicine and Hygiene, vol. 79, no. 5, pp. 719-721, 1985.

[24] A. Belay, M. Ashagrie, B. Seyoum, M. Alemu, and A. Tsegaye, "Prevalence of enteric pathogens, intestinal parasites and resistance profile of bacterial isolates among HIV infected and noninfected diarrheic patients in Dessie Town, Northeast Ethiopia," PLOS ONE, vol. 15, no. 12, article e0243479, 2020.

[25] G. Tadesse, "Prevalence of human Salmonellosis in Ethiopia: a systematic review and meta-analysis," BMC infectious diseases, vol. 14, no. 1, p. 88, 2014

[26] T. Eguale, W. A. Gebreyes, D. Asrat, H. Alemayehu, J. S. Gunn, and E. Engidawork, "Non-typhoidal Salmonella serotypes, antimicrobial resistance and co-infection with parasites among patients with diarrhea and other gastrointestinal complaints in Addis Ababa, Ethiopia," BMC Infectious Diseases, vol. 15, no. 1, p. 497, 2015.

[27] G. Beyene, S. Nair, D. Asrat, Y. Mengistu, H. Engers, and J. Wain, "Multidrug resistant Salmonella Concord is a major cause of salmonellosis in children in Ethiopia," Journal of Infection in Developing Countries, vol. 5, no. 1, pp. 23-33, 2011.

[28] K. Abera, T. L. Anticho, and M. M. Ali, "Salmonella and Shigella and antimicrobial susceptibility profiles among adult patients with complaints of diarrhea at Hawassa comprehensive specialized hospital, Hawassa, Ethiopia," SAGE open medicine, vol. 9, 2021.

[29] A. Gebre-Yohannes, "Salmonella from Ethiopia: prevalent species and their susceptibility to drugs," Ethiopian Medical Journal, vol. 23, no. 3, pp. 97-102, 1985.

[30] S. Kariuki, G. Revathi, N. Kariuki, J. Kiiru, J. Mwituria, and C. A. Hart, "Characterisation of community acquired nontyphoidal Salmonella from bacteraemia and diarrhoeal infections in children admitted to hospital in Nairobi, Kenya," BMC Microbiology, vol. 6, no. 1, p. 101, 2006.

[31] O. Lunguya, V. Lejon, M. F. Phoba et al., "Antimicrobial resistance in invasive non-typhoid Salmonella from the Democratic 
Republic of the Congo: emergence of decreased fluoroquinolone susceptibility and extended-spectrum beta lactamases," PLoS neglected tropical diseases, vol. 7, no. 3, article e2103, 2013.

[32] R. Lan, P. R. Reeves, and S. Octavia, "Population structure, origins and evolution of major Salmonella enterica clones," Infection, Genetics and Evolution, vol. 9, no. 5, pp. 996-1005, 2009.

[33] M. Tesfaye, Study on Knowledge, Attitudes and Behavioral Practices of Animal and Human Antimicrobial Usage and Antimicrobial Resistance amongst People in Bishoftu Town, Ethiopia, Department of Clinical Studies, College of Veterinary Medicine and Agriculture, Ed., Addis Ababa University, Bishoftu, 2017.

[34] M. Haenssgen, T. Xayavong, N. Charoenboon, P. Warapikuptanun, and Y. Zaw, "The consequences of AMR education and awareness raising: outputs, outcomes, and behavioural impacts of an antibiotic-related educational activity in Lao PDR," Antibiotics, vol. 7, no. 4, p. 95, 2018.

[35] J. A. Ayukekbong, M. Ntemgwa, and A. N. Atabe, "The threat of antimicrobial resistance in developing countries: causes and control strategies," Antimicrobial Resistance and Infection Control, vol. 6, no. 1, p. 47, 2017.

[36] S. Maraki and I. S. Papadakis, "Serotypes and antimicrobial resistance of human nontyphoidal isolates of Salmonella enterica from Crete, Greece," Interdisciplinary perspectives on infectious diseases, vol. 2014, Article ID 256181, 5 pages, 2014.

[37] I. A. Altayyar, M. F. Elbreki, M. O. Ali, and A. A. Ali, "Prevalence and antimicrobial susceptibility patterns of Salmonella spp isolated from gastroenteritis patients, Southwestern, Libya," Journal of Applied Medical and Biomedical Research, vol. 1, no. 2, pp. 2-6, 2016.

[38] S. Neuert, S. Nair, M. R. Day et al., "Prediction of phenotypic antimicrobial resistance profiles from whole genome sequences of non-typhoidal Salmonella enterica," Frontiers in Microbiology, vol. 9, pp. 592-592, 2018.

[39] D. Asrat, "Shigella and Salmonella serogroups and their antibiotic susceptibility patterns in Ethiopia," EMHJ - Eastern Mediterranean Health Journal, vol. 14, no. 4, pp. 760-767, 2008.

[40] M. Mama and G. Alemu, "Prevalence, antimicrobial susceptibility patterns and associated risk factors of Shigella and Salmonella among food handlers in Arba Minch University, South Ethiopia," BMC infectious diseases, vol. 16, no. 1, p. 686, 2016.

[41] X. Wang, S. Biswas, N. Paudyal et al., "Antibiotic resistance in Salmonella Typhimurium isolates recovered from the food chain through National Antimicrobial Resistance Monitoring System between 1996 and 2016," Frontiers in Microbiology, vol. 10, p. 985, 2019. 